28. Hahnloser, R. H., Sarpeshkar, R., Mahowald, M. A., Douglas, R. J. \& Seung, H. S. Digital selection and analogue amplification coexist in a cortex-inspired silicon circuit. Nature 405, 947-951 (2000) 29. Stern, P., Edwards, F. A. \& Sakmann, B. Fast and slow components of unitary EPSCs on stellate cells elicited by focal stimulation in slices of rat visual cortex. J. Physiol. (Lond.) 449, 247-278 (1992).

30. Connors, B. W., Malenka, R. C. \& Silva, L. R. Two inhibitory postsynaptic potentials, and $\mathrm{GABA}_{\mathrm{A}}$ and $\mathrm{GABA}_{\mathrm{B}}$ receptor-mediated responses in neocortex of rat and cat. J. Physiol. (Lond.) 406, 443-468 (1988).

Supplementary Information accompanies the paper on www.nature.com/nature

Acknowledgements We thank R. Yuste for his comments. This work was supported by the National Institutes of Health (D.A.M.), the Human Frontier Science Program (D.A.M.), and by a fellowship from the Howard Hughes Institute (A.H.).

Competing interests statement The authors declare that they have no competing financial interests.

Correspondence and requests for materials should be addressed to D.A.M. (david.mccormick@yale.edu).

\section{Recurrent de novo point mutations in lamin A cause Hutchinson-Gilford progeria syndrome}

Maria Eriksson*, W. Ted Brown $\dagger$, Leslie B. Gordon $\neq$, Michael W. Glynn $\$$, Joel Singer\|, Laura Scott||, Michael R. Erdos, Christiane M. Robbins*, Tracy Y. Moses*, Peter Berglund ๆ, Amalia Dutra*, Evgenia Pak*, Sandra Durkin $\$$, Antonei B. Csoka\#, Michael Boehnke\|, Thomas W. Glover $\$$ \& Francis S. Collins ${ }^{\star}$

* National Human Genome Research Institute, and $\|$ Laboratory of Viral Diseases, National Institute of Allergy and Infectious Diseases, National Institutes of Health, Bethesda, Maryland 20892, USA

$\dagger$ Department of Human Genetics, New York State Institute for Basic Research in Developmental Disabilities, Staten Island, New York 10314, USA $\ddagger$ Department of Anatomy and Cellular Biology, Tufts University School of Medicine, Boston, Massachusetts 02111, and Department of Pediatrics, Rhode Island Hospital, Providence, Rhode Island 02903, USA

$\$$ Department of Human Genetics, and $\|$ Department of Biostatistics, University of Michigan, Ann Arbor, Michigan 48109, USA

\# Department of Molecular Biology, Cell Biology, and Biochemistry, Brown University, Providence, Rhode Island 02912, USA

Hutchinson-Gilford progeria syndrome (HGPS) is a rare genetic disorder characterized by features reminiscent of marked premature ageing ${ }^{1,2}$. Here, we present evidence of mutations in lamin A (LMNA) as the cause of this disorder. The HGPS gene was initially localized to chromosome 1q by observing two cases of uniparental isodisomy of 1q-the inheritance of both copies of this material from one parent-and one case with a 6-megabase paternal interstitial deletion. Sequencing of $L M N A$, located in this interval and previously implicated in several other heritable disorders $^{3,4}$, revealed that 18 out of 20 classical cases of HGPS harboured an identical de novo (that is, newly arisen and not inherited) single-base substitution, G608G(GGC > GGT), within exon 11. One additional case was identified with a different substitution within the same codon. Both of these mutations result in activation of a cryptic splice site within exon 11, resulting in production of a protein product that deletes $\mathbf{5 0}$ amino acids near the carboxy terminus. Immunofluorescence of HGPS fibroblasts with antibodies directed against lamin A revealed that many cells show visible abnormalities of the nuclear membrane. The discovery of the molecular basis of this disease may shed light on the general phenomenon of human ageing.

Premature senescence is the hallmark of HGPS (Online Mende- lian Inheritance in Man (OMIM) 176670), both clinically and in cell culture $^{1,2}$. Children affected with HGPS typically appear normal at birth, but within a year the characteristic features of failure to thrive, delayed dentition, alopecia and sclerodermatous skin changes begin to appear. Death occurs on average at age 13, and at least $90 \%$ of the patients die from progressive atherosclerosis of the coronary and cerebrovascular arteries ${ }^{2}$. Although the prevailing hypothesis of the inheritance of HGPS has been sporadic autosomal dominant ${ }^{5,6}$, the possibility of autosomal recessive inheritance ${ }^{7,8}$ led us to conduct a genome-wide scan searching for evidence of homozygosity 9 . A whole-genome scan including 403 polymorphic microsatellite markers with an average spacing of $9.2 \mathrm{cM}$ was performed on 12 DNA samples derived from individuals considered by one of us (W.T.B.) to represent classical HGPS. Although no evidence of homozygosity was identified in the overall sample set, two HGPS samples were found to have uniparental isodisomy (UPD) of chromosome 1q (Fig. 1a). Spectral karyotyping and G-banding of one of the two UPD cases showed a normal karyotype (data not shown).

An earlier report ${ }^{10}$ described an abnormal karyotype in a monozygotic twin with HGPS. That report described a mosaic cell population in which $70 \%$ of the cells contained a balanced inverted insertion $(46, \mathrm{XY}$, inv ins $(1 ; 1)(\mathrm{q} 32 ; \mathrm{q} 44 \mathrm{q} 23))$, whereas the rest of the cells had an apparently normal karyotype. We obtained a fibroblast culture from the same individual (sample identification C8803), as well as his parents. Unexpectedly, genotyping of microsatellite markers identified a roughly 6-megabase $(\mathrm{Mb})$ interval where all tested paternal alleles were completely missing (Fig. 1b). We confirmed that this deletion was also present in the cells that had an apparently normal karyotype, using fluorescent in situ hybridization (FISH) with bacterial artificial chromosomes (BACs) that map throughout this interval (Fig. 1c). Putting all of this information together with genotypes from a total of 44 additional microsatellite markers, we determined that the HGPS gene must lie in an interval of $4.82 \mathrm{Mb}$ on proximal chromosome 1q (Fig. 1d).

The candidate interval contains roughly 80 known genes. Our attention was immediately drawn to one of them, the LMNA gene that encodes two protein products (lamin A and lamin C), representing major constituents of the inner nuclear membrane lamina. Mutations in LMNA have been found to be the cause of six different recessive and dominant disorders, including Emery-Dreifuss muscular dystrophy type 2, a form of dilated cardiomyopathy, the Dunnigan type of familial partial lipodystrophy, limb girdle muscular dystrophy type 1B, Charcot-Marie-Tooth disorder type 2B1, and mandibuloacral dysplasia (see reviews of laminopathies in refs $3,4)$. The gene contains 12 exons and covers about 25 kilobases of genomic DNA. Lamin A is coded by exons 1-12 and lamin C by exons 1-10. A splice site within exon 10, located just upstream of the stop codon for lamin $\mathrm{C}$, splices together with exons 11 and 12 to code for lamin $\mathrm{A}^{11,12}$.

Polymerase chain reaction (PCR) amplification of all of the exons of the LMNA gene (including exon-intron boundaries), followed by direct sequencing, was carried out in 23 samples from patients with classical HGPS. In 18 of these samples a heterozygous base substitution (G608G(GGC > GGT)) within exon 11 of the LMNA gene was identified (Fig. 2a). In HGPS sample AG10801, a different heterozygous base substitution was identified within the same codon (G608S(GGC > AGC)) (Fig. 2a). In HGPS sample AG10677, we identified a heterozygous base substitution within exon $2(\mathrm{E} 145 \mathrm{~K}(\mathrm{GAG}>\mathrm{AAG}))$. In the eight cases where DNA from both parents was available, the G608G mutation was absent in the parents, confirming that these are de novo mutations. Similarly, the G608S and E145K mutations were not found in parents of AG10801 or AG10677, respectively. Thus, of the 23 classical HGPS cases studied, there were only three in which no LMNA mutations were found (Table 1): the two UPD cases (AG10578 and HGADFN005) and the sample with the $6-\mathrm{Mb}$ paternal deletion $(\mathrm{C} 8803)$. 
The de novo recurrence of the same exact point mutation in 18 out of 20 cases of classical HGPS is a surprising finding, but is not without precedent. The common HGPS mutation is a $\mathrm{C}$ to $\mathrm{T}$ in the context of a CpG dinucleotide, which represents the most mutable base in the vertebrate genome, as a methylated $\mathrm{C}$ can be deaminated to $\mathrm{T}$ and miscopied. In the $\mathrm{E} 145 \mathrm{~K}$ case and in four out of four G608G HGPS cases where parental DNA was available and silent polymorphisms in exon $10(\mathrm{H} 566 \mathrm{H})$ or exon 7 (D446D) were informative, we were able to determine that the new mutation was paternal in origin (data not shown). A very similar phenomenon occurs in achondroplasia ${ }^{13}$, where nearly all sporadic cases are due to paternal $\mathrm{CpG}$ to TpG mutations in the FGFR3 gene, resulting in an apparent gain-of-function mutation.

The most common mutation, G608G(GGC > GGT), is a silent substitution. The second mutation in the same codon,
G608S(GGC > AGC), results in a conservative substitution of serine for glycine. How is it possible that these bland-appearing de novo mutations could cause HGPS? Inspection of the normal sequence surrounding codon 608 reveals that both of the observed HGPS mutations improve the match to a consensus splice donor (Fig. 2b), suggesting that they might activate a cryptic splice site. To test this hypothesis, PCR with reverse transcription (RT) was performed using a forward primer spanning the junction of exons 7 and 8 , and a reverse primer in exon 12. In RNA from unaffected individuals, the expected product appears (Fig. 2c). In RNA samples from cell lines harbouring HGPS mutations, an additional smaller RT-PCR product appears. Sequence of these fragments shows that 150 nucleotides within exon 11 are missing. As the reading frame is maintained, this abnormal transcript would be expected to code for a protein with an internal deletion of 50 amino acids near the $\mathrm{C}$ a

46,XY UPD (1)(p11.2;qter)
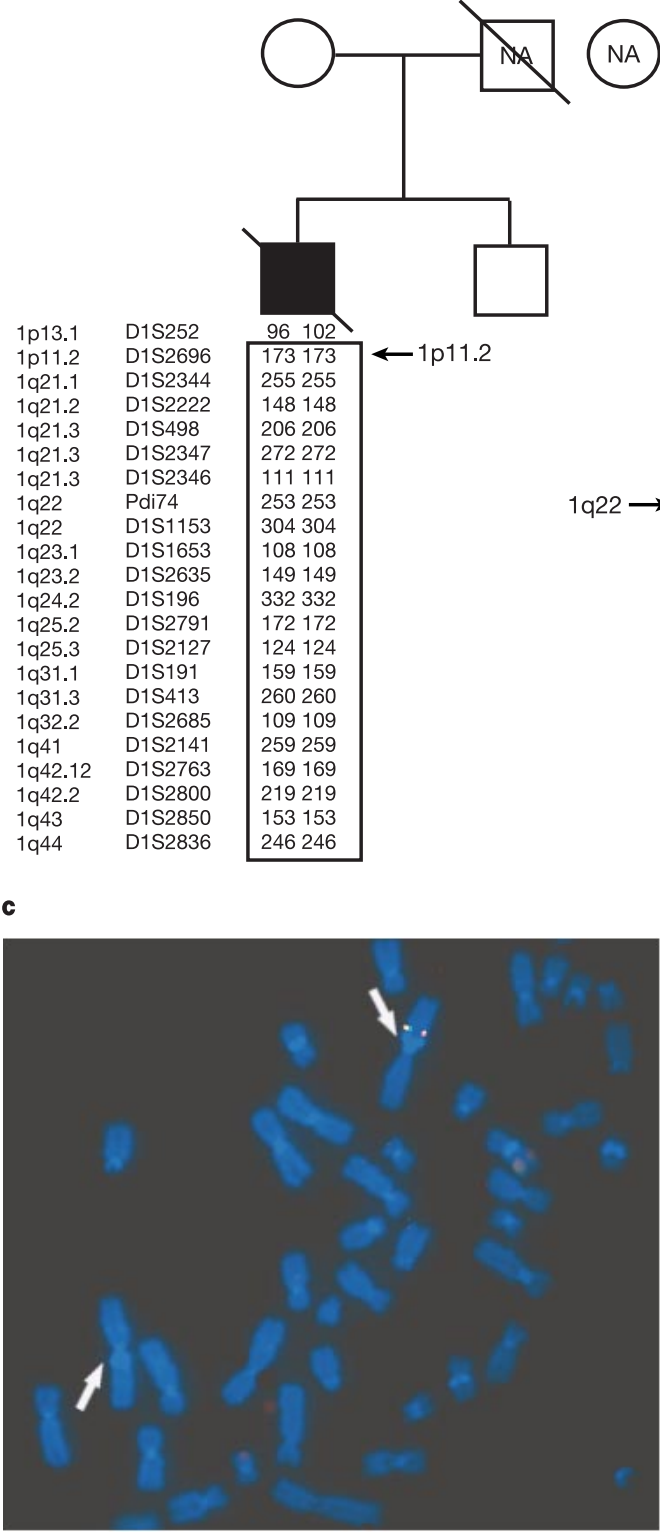

46,XX UPD (1)(q22;qter)

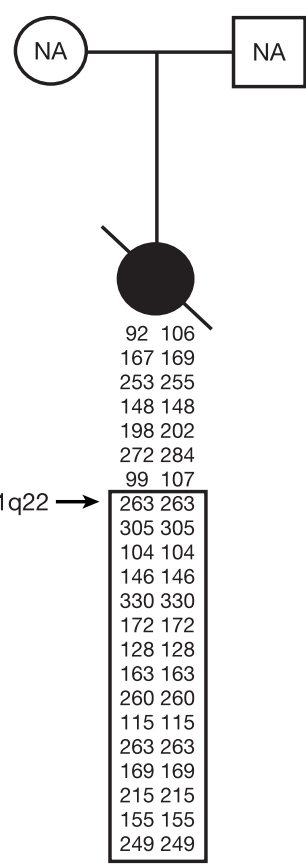

b
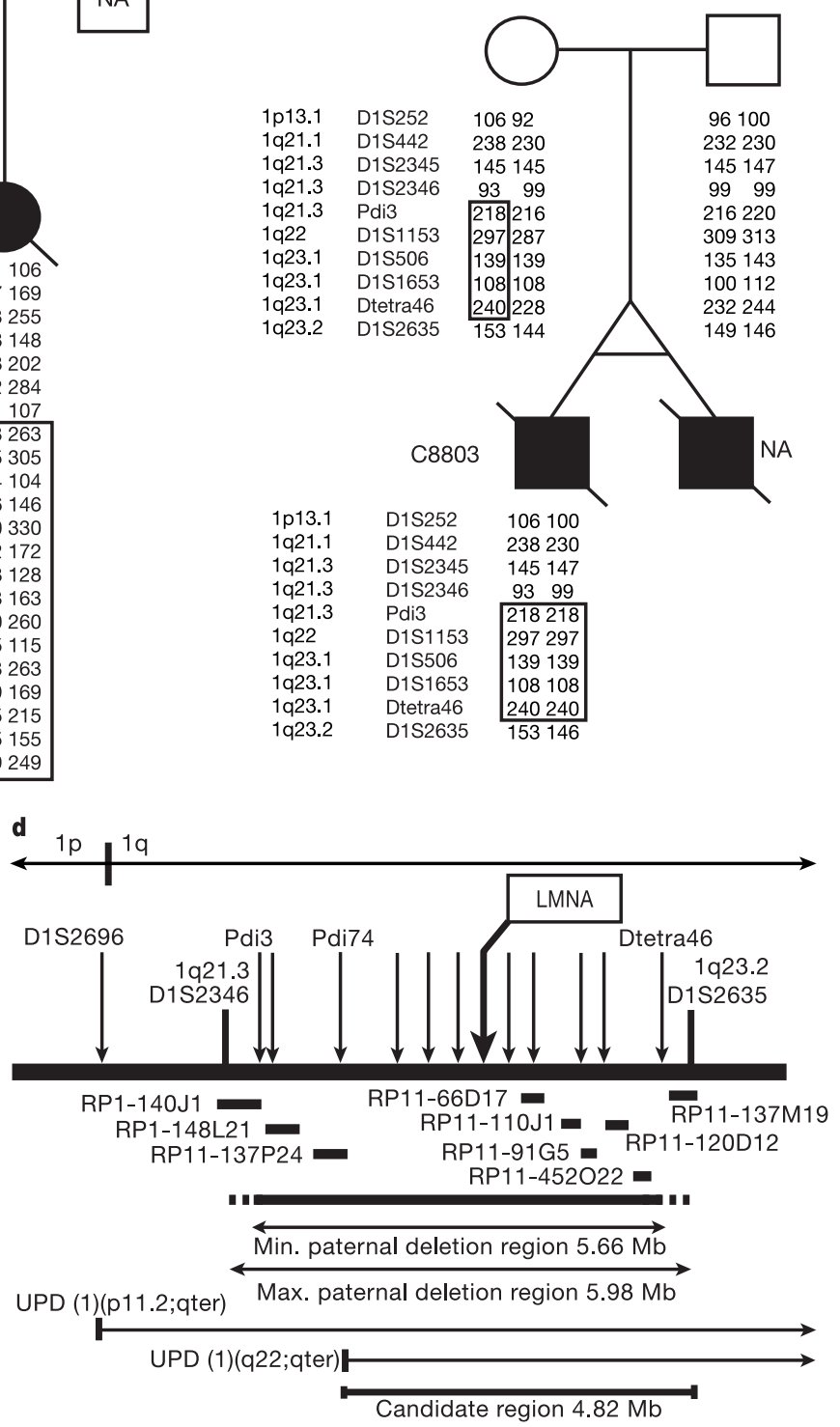

hybridized with BAC probes RP11-110J1 (green) and RP11-91G5 (red). d, Summary map of the candidate region. Microsatellite markers are indicated with arrows; horizontal bars indicate BAC probes that were used for FISH on sample C8803. LMNA is one of the approximately 80 known genes in the 4.82-Mb candidate interval.
Figure 1 Localization of the HGPS gene to a 4.82-Mb interval on chromosome 1q. a, Uniparental isodisomy of chromosome $1 q$ in fibroblasts from two patients with HGPS (filled symbols). A subset of markers and their genotypes are shown. NA, not available. b. The boxed interval is the region in proband $\mathrm{C} 8803$ that has been inherited exclusively from the mother. c, FISH analysis of a metaphase spread from C8803 fibroblasts, 
terminus of lamin A. Lamin C would be unaffected. Cloning and sequencing of the normal full-length fragment obtained from RT-PCR on RNA extracted from primary fibroblasts containing the more common codon 608 mutation revealed that 7 out of 23 clones carry the mutant sequence. Thus, activation of the cryptic

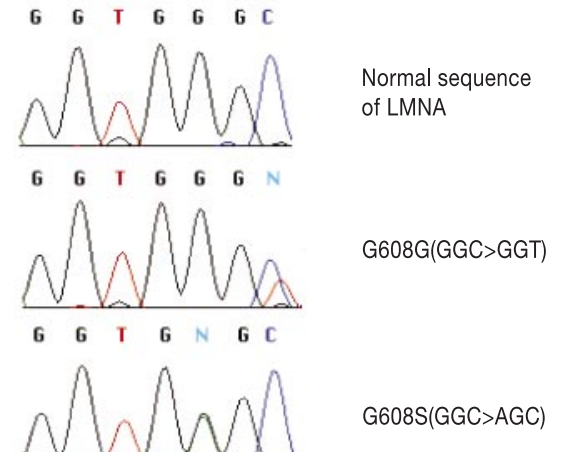

b

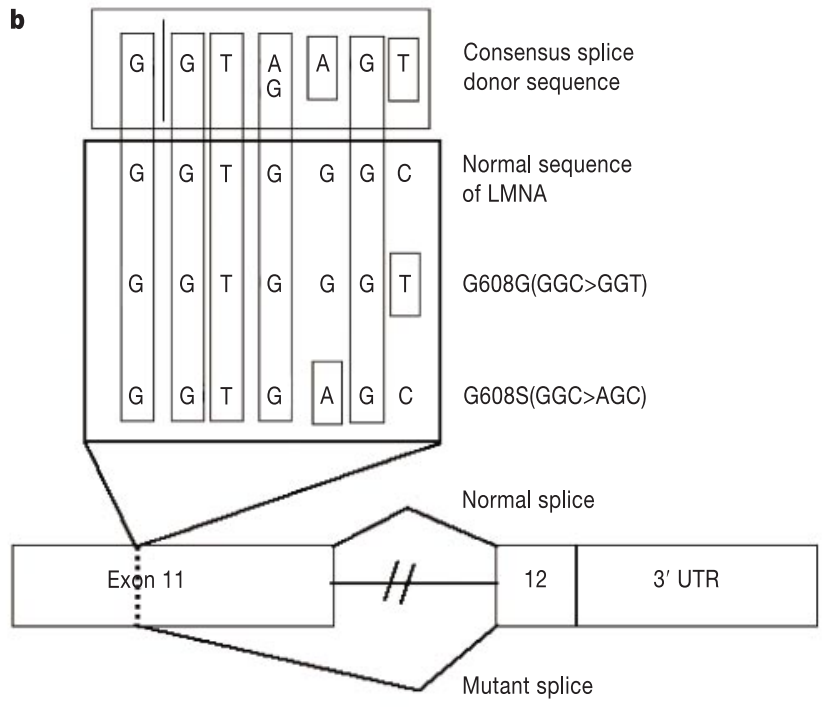

c

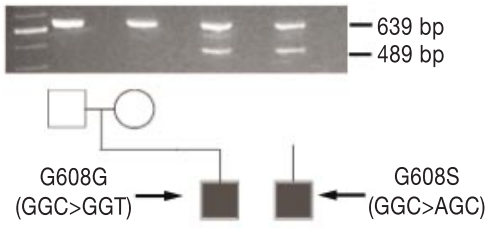

d splice site within exon 11 is not complete. To determine whether the mutant messenger RNA is actually translated into protein, a western blot was performed using a monoclonal antibody against lamin $\mathrm{A} / \mathrm{C}$ (Fig. 2d). In addition to the normal bands for lamin A and lamin C, an additional band is present in four of the lanes corresponding to samples from classical HGPS cases, but not in their parents. The abnormal band is not visible in the lane that contains the protein sample from classical HGPS patient AG11498 (which carries G608G(GGC > GGT)), but this is probably due to the very small amount of lamin A being expressed in this particular fibroblast line.

Although the major cause of HGPS appears to be the creation of an abnormal splice donor in exon 11, the finding of a de novo point mutation in exon 2 in a single patient (AG10677) is of interest. In retrospect, this patient ${ }^{14}$ had atypical clinical features (including persistence of coarse hair over the head, ample subcutaneous tissue over the arms and legs, and severe strokes beginning at age 4) that may subtly distinguish this phenotype from classical HGPS.

The two UPD cases present an interesting dilemma: if the LMNA gene sequence is normal in both cases, why do they have HGPS? The possibility of imprinting must be considered; however, previous cases of both paternal and maternal complete isodisomy of chromosome 1 do not support the presence of any imprinted loci on this chromosome $\mathrm{e}^{15,16}$. In the case where DNA samples were available from the mother and sibling (Fig. 1a), we can conclude that this phenomenon resulted in a chromosome that has partly paternal and partly maternal alleles. This must have arisen by a post-fertilization event, probably a mitotic crossover between homologues. We postulate that these cases actually represent 'somatic rescue' events of the premature senescence phenotype of HGPS. Under this hypothesis, the individuals from whom these fibroblasts were derived originally harboured typical codon 608 HGPS mutations in LMNA. Perhaps as an in vivo event, or perhaps as an in vitro event in the fibroblast culture, a mitotic crossover occurred, generating a cell with segmental UPD that had duplicated the wild-type allele of LMNA and lost the HGPS mutation. Such a cell would probably then have a growth advantage over its neighbours. Proof of this hypothesis would require access to multiple tissues of the deceased UPD patients, which unfortunately are not available.

We also did not identify any mutation in the LMNA gene in the patient with the 6-Mb deletion (Fig. 1b). We believe that this might also be due to a somatic rescue event-specifically, we hypothesize that this patient was originally heterozygous for a codon 608 LMNA mutation, but in this instance the 'rescue' involved an internal deletion of $6 \mathrm{Mb}$ containing the mutant allele, associated with a more complex mosaic rearrangement of chromosome 1. A recent study reported on a case of classical HGPS in which an apparent interstitial deletion of chromosome 1q23 was seen ${ }^{17}$. We obtained cells and DNA from this patient, and to our surprise the typical heterozygous G608G mutation is present (data not shown). Furthermore, we have not been able to confirm the presence of an interstitial deletion by high-resolution chromosome banding or by FISH with BACs spanning the 1q23-1q24 region. This may be another example of somatic rescue, involving an interstitial deletion in the clone of cells analysed in the original report, but not present in other samples from the same patient.

Extensive prior study of the biochemical function of lamin A suggests a possible mechanism for disease causation. Lamin A is normally synthesized as a precursor molecule (prelamin A). At the $\mathrm{C}$ terminus is a CAAX-box motif that is subject to farnesylation. After that, an internal proteolytic cleavage occurs, removing the last 18 coding amino acids ${ }^{18-20}$, to generate mature lamin A. We predict that the codon 11 HGPS mutations and consequent abnormal splicing would produce a prelamin A that still retains the CAAX box, but is missing the site for endoproteolytic cleavage. There is also evidence that cell-cycle-dependent phosphorylation of lamin A is important for its normal function, and at least one site for phosphorylation (Ser 625) is deleted in the abnormal HGPS 


\begin{tabular}{|c|c|c|c|c|c|c|}
\hline \multicolumn{7}{|c|}{ Table 1 LMNA mutations in samples from classical HGPS } \\
\hline \multirow[b]{2}{*}{ Sample ID (classical HGPS) } & \multirow[b]{2}{*}{ Codon 608 nucleotide sequence } & \multirow[b]{2}{*}{ Mutation } & \multirow[b]{2}{*}{ Comment } & \multicolumn{3}{|c|}{ Codon 608 nucleotide sequence } \\
\hline & & & & Mother & Father & Sibling(s) \\
\hline AG01972 & GGC/T & G608G & - & NA & NA & NA \\
\hline AG06297 & $\mathrm{GGC} / \mathrm{T}$ & G608G & - & NA & NA & NA \\
\hline AG11498 & $\mathrm{GGC} / \mathrm{T}$ & G608G & - & NA & NA & NA \\
\hline AG11513 & $\mathrm{GGC} / \mathrm{T}$ & G608G & - & NA & NA & NA \\
\hline AG03506 & $\mathrm{GGC} / \mathrm{T}$ & G608G & - & Normal & Normal & Normal \\
\hline AG03344 & $\mathrm{GGC} / \mathrm{T}$ & G608G & - & Normal & Normal & Normal \\
\hline AG03259 & $\mathrm{GGC} / \mathrm{T}$ & G608G & - & Normal & Normal & Normal \\
\hline AG06917 & $\mathrm{GGC} / \mathrm{T}$ & G608G & - & Normal & Normal & NA \\
\hline AG10579 & $\mathrm{GGC} / \mathrm{T}$ & G608G & - & NA & NA & NA \\
\hline AG10587 & $\mathrm{GGC} / \mathrm{T}$ & G608G & - & Normal & NA & Normal \\
\hline HGADFN001 & GGC/T & G608G & - & NA & NA & NA \\
\hline HGADFN003 & GGC/T & G608G & - & NA & NA & NA \\
\hline HGALBV009 & GGC/T & G608G & - & Normal & Normal & NA \\
\hline HGALBV011 & $\mathrm{GGC} / \mathrm{T}$ & G608G & - & Normal & Normal & NA \\
\hline HGALBV057 & GGC/T & G608G & - & Normal & Normal & NA \\
\hline HGADFN008 & $\mathrm{GGC} / \mathrm{T}$ & G608G & - & NA & NA & NA \\
\hline HGADFN014 & $\mathrm{GGC} / \mathrm{T}$ & G608G & - & NA & NA & NA \\
\hline HGALBV071 & $\mathrm{GGC} / \mathrm{T}$ & G608G & - & NA & NA & NA \\
\hline AG10801 & A/GGC & G608S & - & Normal & Normal & NA \\
\hline AG10578 & GGC & - & UPD & Normal & NA & Normal \\
\hline HGADFN005 & GGC & - & UPD & NA & NA & NA \\
\hline C8803 & GGC & - & Deletion & Normal & Normal & NA \\
\hline AG10677 & GGC & E145K & - & Normal $^{*}$ & Normal $^{*}$ & NA \\
\hline
\end{tabular}

protein $^{21}$. As lamin A forms a multiprotein complex within the inner nuclear membrane, this incompletely processed prelamin A might well act as a dominant negative.

Notably, defective prelamin A processing has recently been identified in a mouse knockout of the Zmpste24 metalloproteinase $\mathrm{e}^{22,23}$. Zmpste24 is believed to be involved in proteolytic processing of prelamin A, and may represent the actual endoprotease. The homozygote knockout of Zmpste24 has a phenotype resembling the clinical features observed in HGPS patients, including growth retardation, premature death (20 weeks) from cardiac dysfunction, and alopecia. However, additional features such as pronounced osteoporosis are also present ${ }^{23}$. Immunofluorescence experiments on cells from these animals show elongated and irregular nuclei with herniation of the nuclear membrane. A mouse knockout of the Lmna gene has also been reported previously ${ }^{24}$, showing severe postnatal growth retardation, muscular dystrophy and similar nuclear abnormalities.

To pursue the possibility of structural nuclear membrane abnormalities in HGPS, immunofluorescence studies with two different monoclonal antibodies against lamin $\mathrm{A} / \mathrm{C}$ were performed on primary fibroblasts from five classical HGPS cases with the common G608G mutation, as well as unaffected parents. Representative data from an affected patient (AG11498) and an unaffected parent (AG06299) are shown in Fig. 3. In 48\% of the HGPS cells we noted an irregular shape of the nuclear envelope (Fig. 3e-h). Cells from the unaffected control (Fig. 3a-d) showed significantly fewer cells of this phenotype $(<6 \%)$. To be certain that this result was not an artefact of secondary differences in the status of the HGPS and control fibroblast cultures, cells originating from the same flasks as the cells used for the immunofluorescence studies
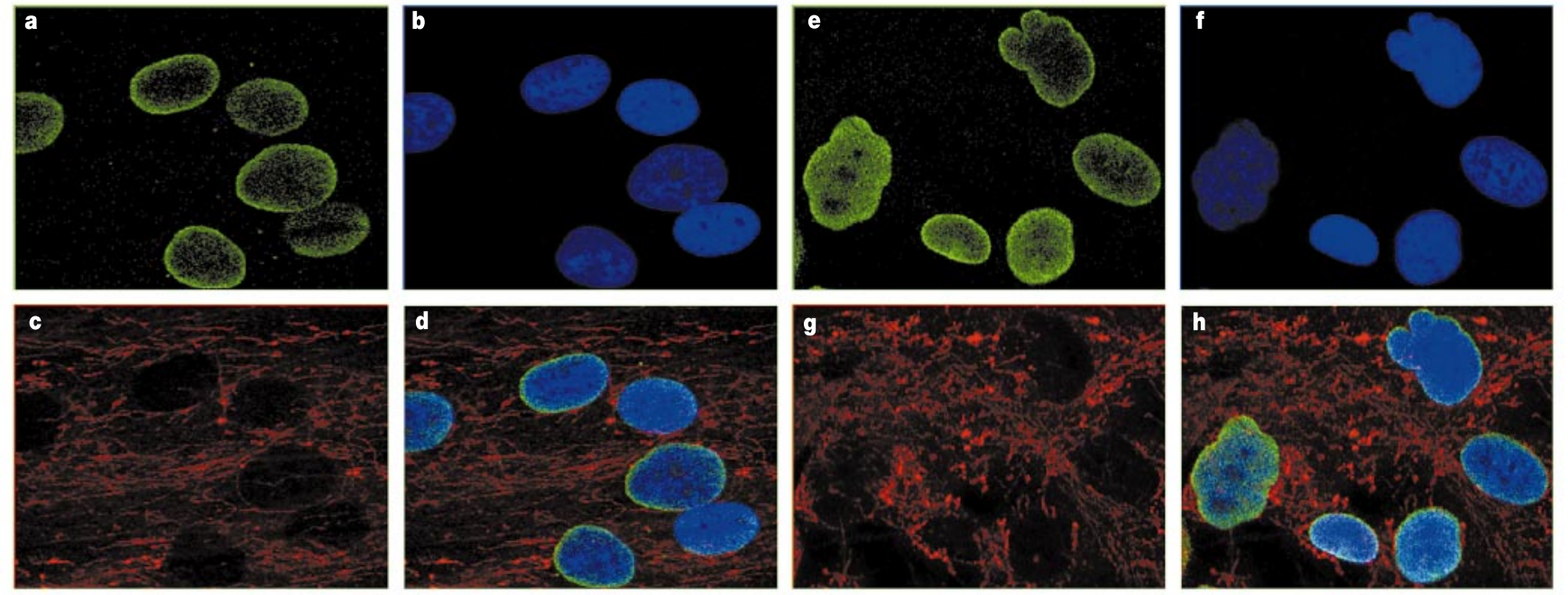

Figure $\mathbf{3}$ Immunofluorescence on primary dermal fibroblasts from an unaffected mother and a child with classical HGPS, using antibody JOL2 against lamin A/C. Identical results were obtained with antibody XB10 (data not shown). a-d, Results from an unaffected mother, AG06299. e-h, Results from a classical HGPS patient, AG11498. a, e, Antibody

against lamin A/C. $\mathbf{b}, \mathbf{f}$, DAPI staining showing location of the nuclei. $\mathbf{c}, \mathbf{g}$, Antibody staining mitochondria, showing distribution of the cytosol. $\mathbf{d}, \mathbf{h}$, Merged image of $\mathbf{a}-\mathbf{c}(\mathbf{d})$ and $\mathbf{e}-\mathbf{g}(\mathbf{h})$. 
were monitored for differences in cell cycle (by fluorescent-activated cell analysis) and degree of apoptosis. No significant differences between the cells derived from the classical HGPS patients and the unaffected parents were noted.

HGPS can now be added to the long list of human genetic disorders shown to be due to mutations in the LMNA gene ${ }^{3,4}$. As most cases of HGPS appear to have a de novo mutation in the same codon, molecular diagnostics should be almost immediately feasible. This will be particularly useful in making the diagnosis in a young child before the full clinical phenotype has appeared. Molecular diagnostic methods may also provide reassurance in the prenatal arena, where the possibility of parental somatic mosaicism and recurrence of disease in future pregnancies can now be addressed. Finally, the discovery of the molecular basis of HGPS suggests a possible role for LMNA in aspects of the normal ageing process. It will be important to look for common variants in this gene that might show association with exceptional longevity, and perhaps also to explore whether somatic mutations in LMNA, accumulated over a lifetime, have some role in senescence.

\section{Methods}

\section{Subjects and DNA/RNA preparation}

This study was approved through the NIH Human Subjects review process. Primary dermal fibroblast cell cultures and EBV-transformed lymphoblastoid cell lines from individuals diagnosed as classical HGPS, and their first-degree relatives (when available), were obtained from the Aging Repository of the Coriell Cell Repository (CCR), and the Progeria Research Foundation Cell and Tissue Bank. DNA was prepared using the Puregene DNA isolation kit (Gentra Systems). The genome scan for homozygosity included samples derived from 12 classical HGPS patients (samples AG01972, AG03259, AG03344, AG03506, AG06297, AG06917, AG10578, AG10579, AG10587, AG10801, AG11498 and AG11513) and 16 unaffected first-degree relatives (samples AG03258, AG03260, AG03262, AG03263, AG03343, AG03342, AG03345, AG03346, AG03504, AG03505, AGO3507, AGO3508, AG06298, AG06299, AG10585 and AG10588). Additional samples used in this study were samples from classical HGPS (samples AG10677, HGADFN001, HGADFN003, HGALBV009, HGALBV011, HGALBV057, HGADFN005, HGADFN008, HGADFN014, HGALBV071 and C8803 (also known as AG10548 in the $\mathrm{CCR})$ ) and samples derived from their unaffected first-degree relatives (samples HGMLBV010, HGFLBV021, HGMLBV023, HGFLBV031, HGMLBV066, HGFLBV067, HGMLBV013, HGFLBV050, HGMLBV058, HGSLBV059, HGMLBV078 HGFLBV079, HGFLBV082 and HGMLBV081). Total RNA was extracted from cells with TRIzol reagent (Invitrogen)

\section{Genotyping}

The whole-genome scan included 403 highly polymorphic microsatellite markers with an average spacing of $9.2 \mathrm{cM}$ and average heterozygosity of about 0.8 (E. Gillanders, M. Jones, D. Freas-Lutz, R. Sood and J. Trent, manuscript in preparation). Pedigree checking was performed using PedCheck (J. O'Connell, http://watson.hgen.pitt.edu/register/docs/ pedcheck.html) and any identified genotype errors were removed. We carried out homozygosity mapping assuming various degrees of inbreeding for the HGPS cases ${ }^{25}$. Additional microsatellite repeats on chromosome 1q were identified using the Sputnik program (C. Abajian, http://rast.abajian.com/sputnik/) (see Supplementary Table 1) and were used to further investigate the UPD cases and the paternal deletion region in C8803. Microsatellite markers were analysed using a 3100 genetic analyser (PE Biosystems). Genotypes were analysed using GeneScan 3.7 and Genotyper 2.5 Software (PE Biosystems).

\section{FISH analysis}

Single- and two-colour FISH was performed on metaphases from sample C8803 following previously published procedures ${ }^{26}$, using a subset of BACs in the region of the paternal deletion. BACs used as probes for the FISH were RP1-140J1, RP1-148L21, RP1-178F15, RP11-137P24, RP11-66D17, RP11-110J1, RP11-91G5, RP11-120D12, RP11-101J8, RP11-81N17, RP11-144L1, RP11-317F9, RP11-452O22 and RP11-137M19.

\section{Mutation analysis of LMNA}

Direct sequencing of $L M N A$ was performed primarily using previously described primer sequences for the LMNA exons 1-12 (ref. 27). Sequencing reactions were performed at quarter strength reaction volumes with the Big Dye Terminator chemistry kit (Applied Biosystems), and electrophoresed on an ABI 3700 DNA Analyser (Applied Biosystems). Multiple sequence alignment was performed with Sequencher (Genecodes Inc.). Attempts were made to sequence all PCR products in both directions, but approximately $7 \%$ of exons failed to yield readable sequence.

\section{RT-PCR on exon 11}

For all RNA samples, $20 \mu \mathrm{g}$ of total RNA was treated with RQ1 RNase-Free DNase according to manufacturers recommendations (Promega). A total of $800 \mathrm{ng}$ of DNasetreated total RNA was used for first-strand complementary DNA synthesis with random hexamers (Superscript, Invitrogen). Control samples without reverse transcriptase were processed at the same time for each sample. PCR primers for the lamin A/C gene were designed in exon $7 / 8$ and exon 12. Primer sequences were $5^{\prime}$-GCAACAAGTCCAATGA GGACCA- $3^{\prime}$ and $5^{\prime}$-GTCCCAGATTACATGATGC-3' ${ }^{\prime}$. PCR fragments were gel-purified or cloned (TOPO TA-cloning kit, Invitrogen) and sequenced. PCR with GAPDH-specific primers was performed on all samples as control.

\section{Western analysis}

Whole cells $\left(1 \times 10^{6}\right)$ were collected and washed twice in PBS, the pellets were resuspended in RIPA buffer and included a cocktail of proteinase inhibitors (Roche). A total of $20 \mu \mathrm{g}$ of protein was loaded and electrophoresed on an $8 \%$ Tris-Glycine mini gel (Invitrogen). Primary monoclonal lamin A/C antibody was clone JOL2 (Chemicon International).

\section{Immunofluorescence}

Cultivated fibroblasts were fixed (3.2\% paraformaldehyde), permeabilized ( $1 \% \mathrm{NP}-40)$, blocked $(0.1 \%$ Brij58 and 5\% serum) and labelled for lamin A/C (monoclonal antibodies JOL2, Chemicon International, and clone XB10, CRP Inc.) mitochondria (HMS-0100, Immunovision Inc.) and DNA (using 4,6-diamidine-2-phenylindole (DAPI) dihydrochloride mixed in at $1 \mu \mathrm{g} \mathrm{ml}^{-1}$ with the second antibody). Analysis was done by confocal microscopy using a Biorad 1024 and Leica SP2 systems.

\section{Cell cycle and apoptosis}

Cells were collected and washed in PBS one day after the immunofluorescence experiments. Duplicate experiments were performed on each cell culture. A total of $5 \times 10^{5}$ cells were resuspended in $0.5 \mathrm{ml}$ of NuCycl propidium iodide (NuCycl PI kit, Exalpha) and processed as recommended by the manufacturer. The total DNA content was measured by DNA flow cytometry. Cells were also assayed for viability using annexin V-fluorescein isothiocyanate and propidium iodide according to standardized procedures (BD Biosciences).

Received 24 January; accepted 1 April 2003; doi:10.1038/nature01629.

Published online 25 April 2003.

1. DeBusk, F. L. The Hutchinson-Gilford progeria syndrome. J. Pediat. 80, 697-724 (1972).

2. Baker, P. B., Baba, N. \& Boesel, C. P. Cardiovascular abnormalities in progeria. Case report and review of the literature. Arch. Pathol. Lab. Med. 105, 384-386 (1981).

3. Burke, B. \& Stewart, C. L. Life at the edge: the nuclear envelope and human disease. Nature Rev. 3, 575-585 (2002).

4. Genschel, J. \& Schmidt, H.-J. Mutations in the LMNA gene encoding lamin A/C. Hum. Mutat. 16, 451-459 (2000).

5. Brown, W. T. Human mutations affecting aging-a review. Mech. Aging Dev. 9, 325-336 (1979).

6. Brown, W. T., Zebrowser, M. \& Kieras, F. J. in Genetic Effects on Aging II (ed. Harrison, D. E.) 521-542 (Telford, Caldwell, New Jersey, 1990).

7. Trevas-Maciel, A. Evidence for autosomal recessive inheritance of progeria (Hutchinson Gilford). Am. J. Med. Genet. 31, 483-487 (1988).

8. Khalifa, M. M. Hutchinson-Gilford progeria syndrome: report of a Libyan family and evidence of autosomal recessive inheritance. Clin. Genet. 35, 125-132 (1989).

9. Lander, E. S. \& Botstein, D. Homozygosity mapping: a way to map human recessive traits with the DNA of inbred children. Science 236, 1567-1570 (1987).

10. Brown, W. T. Progeria: a human disease model of accelerated aging. Am. J. Clin. Nutr. 55, 1222S-1224S (1992).

11. Fisher, D. Z., Chaudhary, N. \& Blobel, G. cDNA sequencing of nuclear lamins A and C reveals primary and secondary structural homology to intermediate filament proteins. Proc. Natl Acad. Sci. USA 83, 6450-6454 (1986).

12. Lin, F. \& Worman, H. J. Structural organization of the human gene encoding nuclear lamin A and nuclear lamin C. J. Biol. Chem. 268, 16321-16326 (1993).

13. Shiang, R. et al. Mutations in the transmembrane domain of FGFR3 cause the most common genetic form of dwarfism, achondroplasia. Cell 78, 335-342 (1994).

14. Smith, A. S., Wiznitzer, M., Karaman, B. A., Horwitz, S. J. \& Lanzieri, C. F. MRA detection of vascular occusion in a child with progeria. Am. J. Neuroradiol. 14, 441-443 (1993).

15. Pulkkinen, L., Bullrich, F., Czarnecki, P., Weiss, L. \& Uitto, J. Maternal uniparental disomy of chromosome 1 with reduction to homozygosity of the LAMB3 locus in a patient with Herlitz junctional epidermolysis bullosa. Am. J. Hum. Genet. 61, 611-619 (1997).

16. Gelb, B. D. et al. Paternal uniparental disomy for chromosome 1 revealed by molecular analysis of a patient with pycnodysostosis. Am. J. Hum. Genet. 62, 848-854 (1998).

17. Delgado-Luengo, W. et al. $\operatorname{Del}(1)$ (q23) in a patient with Hutchinson-Gilford progeria. Am. J. Med. Genet. 113, 298-301 (2002).

18. Lutz, R. J., Trujillo, M. A., Denham, K. S., Wenger, L. \& Sinensky, M. Nucleoplasmic localization of prelamin A: implications for prenylation-dependent lamin A assembly into the nuclear lamina. Proc. Natl Acad. Sci. USA 89, 3000-3004 (1992).

19. Sinensky, M. et al. The processing pathway of prelamin A. J. Cell Sci. 107, 61-67 (1994).

20. Hennekes, H. \& Nigg, E. A. The role of isoprenylation in membrane attachment of nuclear lamins, a single point mutation prevents the proteolytic cleavage of the lamin A precursor and confers membrane binding properties. J. Cell Sci. 107, 1019-1029 (1994).

21. Eggert, M. et al. Identification of novel phosphorylation sites in murine A-type lamins. Eur. J. Biochem. 213, 659-671 (1993).

22. Pendas, A. M. et al. Defective prelamin A processing and muscular and adipocyte alterations in Zmpste24 metalloproteinase-deficient mice. Nature Genet. 31, 94-99 (2002).

23. Bergo, M. O. et al. Zmpste24 deficiency in mice causes spontaneous bone fractures, muscle weakness, and a prelamin A processing defect. Proc. Natl Acad. Sci. USA 99, 13049-13054 (2002).

24. Sullivan, T. et al. Loss of A-type lamin expression compromises nuclear envelope integrity leading to muscular dystrophy. J. Cell Biol. 147, 913-920 (1999).

25. Smith, C. The detection of linkage in human genetics. J. R. Stat. Soc. B 15, 153-184 (1953).

26. Casper, A. M., Nghiem, P., Arlt, M. F. \& Glover, T. W. ATR regulates fragile site stability. Cell 111, 779-789 (2002). 
27. De Sandre-Giovannoli, A. et al. Homozygous defects in $L M N A$, encoding lamin A/C nuclear-envelope proteins, cause autosomal recessive axonal neuropathy in human (Charcot-Marie-Tooth disorder type 2) and mouse. Am. J. Hum. Genet. 70, 726-736 (2002).

28. Speckman, R. A. et al. Mutational and haplotype analyses of families with familial partial lipodystrophy (Dunnigan variety) reveal recurrent missense mutations in the globular C-terminal domain of lamin A/C. Am. J. Hum. Genet. 66, 1192-1198 (2000); erratum 67, 775 (2000).

Supplementary Information accompanies the paper on www.nature.com/nature.

Acknowledgements We are grateful to all of the families with HGPS who donated blood and skin samples, without which this study would not have been possible. We would also like to express our gratitude to the entire Progeria Research Consortium, especially B. Toole, for their encouragement during the course of this work. We also thank E. Gillanders for providing the linkage marker set; P. Hollstein for assistance with genotyping; P. Chines for the identification of additional microsatellite markers on chromosome 1q; and M. Kirby for assistance with the flow cytometry. Support from the Progeria Research Foundation (L.B.G., M.W.G. and T.W.G.) and the Bedminster Foundation (W.T.B.) is gratefully acknowledged.

Competing interests statement The authors declare that they have no competing financial interests.

Correspondence and requests for materials should be addressed to F.S.C. (fc23a@nih.gov).

\section{A progeroid syndrome in} mice is caused by defects in A-type lamins

Leslie C. Mounkes, Serguei Kozlov, Lidia Hernandez, Teresa Sullivan \& Colin L. Stewart

Cancer and Developmental Biology Laboratory, National Cancer Institute, Frederick, Maryland 21702, USA

Numerous studies of the underlying causes of ageing have been attempted by examining diseases associated with premature ageing, such as Werner's syndrome and Hutchinson-Gilford progeria syndrome (HGPS). HGPS is a rare genetic disorder resulting in phenotypes suggestive of accelerated ageing, including shortened stature, craniofacial disproportion, very thin skin, alopecia and osteoporosis, with death in the early teens predominantly due to atherosclerosis ${ }^{1}$. However, recent reports suggest that developmental abnormalities may also be important in HGPS $^{1,2}$. Here we describe the derivation of mice carrying an autosomal recessive mutation in the lamin A gene (Lmna) encoding A-type lamins, major components of the nuclear lamina ${ }^{3}$. Homozygous mice display defects consistent with HGPS, including a marked reduction in growth rate and death by 4 weeks of age. Pathologies in bone, muscle and skin are also consistent with progeria. The Lmna mutation resulted in nuclear morphology defects and decreased lifespan of homozygous fibroblasts, suggesting premature cell death. Here we present a mouse model for progeria that may elucidate mechanisms of ageing and development in certain tissue types, especially those developing from the mesenchymal cell lineage.

The Lmna gene encodes the A-type lamins, intermediate filament family members that make up the nuclear lamina, a fibrous network underlying the nuclear envelope ${ }^{3}$. Alternative splicing of Lmna transcripts gives rise to four proteins: two major products called lamin $\mathrm{A}$ and lamin $\mathrm{C}$, and two minor products, laminA $\Delta 10$ and lamin C2, the latter being specific to the testis. The A-type lamins interact with B-type lamins, which are encoded by the Lmnb1 and $L m n b 2$ genes, in a largely uncharacterized assembly process to form the nuclear lamina. The A-type lamins are developmentally regulated, whereas at least one B-type lamin is expressed in every cell type at all developmental stages. Embryonic stem cells, cells of the early embryo and haematopoietic stem cells do not express Lmna; however, derivatives of these cells do express Lmna during development, suggesting a role for Lmna in terminal differentiation ${ }^{4}$. In addition to controlling interphase nuclear morphology, the nuclear lamina also functions in selective retention of proteins in the inner nuclear membrane, chromatin organization, DNA replication and gene expression ${ }^{5,6}$. A role for A-type lamins in regulating gene expression is further supported by interactions with a growing number of transcription factors, for example, $\mathrm{Rb}^{7}$ and GCL indirectly through emerin ${ }^{8}$.

The indispensable role of lamins in these diverse and fundamental processes may account for their association with a growing list of human diseases. Laminopathies can be divided loosely into two categories: those affecting striated muscle and those with phenotypes affecting adipose tissue and bone. Diseases of striated muscle caused by mutations in the Lmna gene are the autosomal dominant form of Emery-Dreifuss muscular dystrophy (AD-EDMD), limb girdle muscular dystrophy 1B (LGMD1B) and dilated cardiomyopathy with conduction system disease (DCM-CD). A single recessive mutation in Lmna has also been implicated in Charcot-Marie-Tooth syndrome type 2B1 (CMT2B1), a peripheral neuropathy with muscle weakness and wasting. Dunnigan's familial partial lipodystrophy (FPLD) and mandibuloacral dysplasia (MAD), disorders primarily resulting in loss and redistribution of white adipose tissue, are also associated with mutations in Lmna. Hyperlipidaemia, insulin resistance and diabetes are common in FPLD and MAD patients, and bone defects in MAD patients include craniofacial abnormalities, osteolysis of terminal digits and hypoplasia of clavicles?. Many clinical observations of MAD patients are also seen in progeria patients ${ }^{10}$, suggesting that the two disorders may be allelic. Although we do not understand why mutations in Lmna cause such a wide array of phenotypes, the continued discovery of phenotypic overlap between some of these diseases indicates that they may represent a spectrum of related disorders rather than separate diseases. The present description of progeroid symptoms associated with Lmna ${ }^{L 530 P / L 530 P}$ mutant
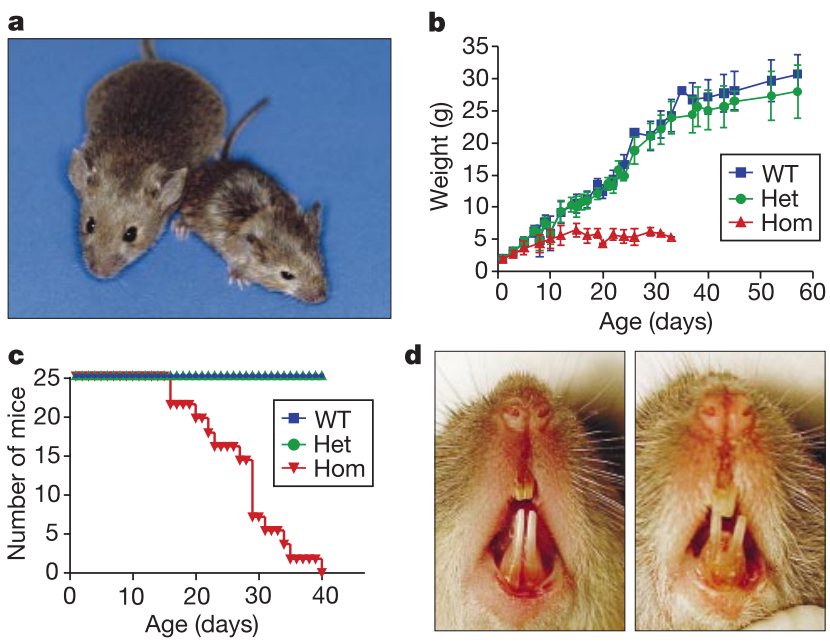

Figure 1 Lmna ${ }^{L 530 P / L 530 P}$ homozygous mice exhibit severely retarded growth and die early. a, The smaller $L m n a^{L 530 P / L 530 P}$ mouse weighed $5.37 \mathrm{~g}$; the littermate $(21.88 \mathrm{~g})$ was wild type. b. Mutants failed to thrive, showing marked growth reduction as early as 4 days after birth. Mice were weighed every $2-3$ days for 58 days. $n=8$ mutant (Hom, red), 10 wild-type (WT, blue) and 14 heterozygotes (Het, green) mice. c, Heterozygotes (green) can live as long as wild-type littermates (blue). Mutants ( $n=25$, red) died by 38 days. d, In $50 \%$ of mutant mice, we observed abnormal dentition in the lower jaw. A heterozygous littermate is on the left 